\title{
A New Approach to Peak Load Forecasting based on EMD and ANFIS
}

\author{
Victor Akpevwe Okolobah and Zuhaimy Ismail* \\ Deparment of Mathematical Sciences, Universiti of Teknologi, Malaysia; dynamic4vic@yahoo.com, \\ zuhaimy@utm.my
}

\begin{abstract}
This paper presents a new approach to Peak Load Forecasting based on EMD and ANFIS. EMD is used to decompose the load data into several Intrinsic Mode Functions (IMFs); then appropriate ANFIS models are developed for these IMFs. The target model proposed in this paper, EMD-ANFIS, is achieved by combining the predictions from these IMF-ANFIS models together and this is used for forecasting purposes. A real life data obtained from Power Holding Company of Nigeria (PHCN), Bida, was used to evaluate the forecast accuracy of the proposed model. The results revealed that the proposed EMD-ANFIS model yields better results when compared to ANN and EMD-ANN models. The proposed EMD-ANFIS model recorded $2.76 \%$ and $50.05 \%$ improvements over EMD-ANN and traditional ANN models, respectively as judged by the overall MAPE of the models.
\end{abstract}

Keywords: EMD, IMF, ANFIS, Forecasting Accuracy, Peak Load.

\section{Introduction}

The current practice in the field of load forecasting research is to combine two or more forecasting tools together. Several such combinations can be observed in the field of load forecasting. This can be seen in many works [1-3] that combined neural networks and fuzzy logic, while some $[4,5]$ combined Support Vector Machine (SVM) and Artificial Neural Networks (ANN) while others [6, 7] combined Genetic Algorithm and Neural Networks in load forecasting. The idea in this is to combine the advantages of these separate methods together and use it to advance the course of the challenge at hand.

Artificial Neural Network (ANN) is an adaptive system which changes its structure during the learning phase. ANN is inspired by biological neurons. The neurons in an ANN are interconnected by information channels which are referred to as interconnections. Each neuron can comprise of several inputs but must have only one output. The inputs to a neuron could be from external sources or from the output of another neuron. There is a connection strength, synapses or weight associated to each connection. To fire a neuron the weighted sum of inputs to the neuron must exceed a given threshold and this produces an output. By tuning or adjusting weights by some learning process, the network recognizes the input patterns. The BackPropagation (BP) Algorithm is a common method for training the feed forward neural network.

Neuro-fuzzy technique is a fusion of ANN and Fuzzy Inference System (FIS) which is very useful in solving real life problems in many fields of human endeavor. The idea behind the neuro-fuzzy system is hinged on fuzzy system that is trained using neural network. In this fusion, the learning capability of ANN is utilized by the FIS, while the rule based linguistic formation of the FIS is used by the ANN [8]. The fusion of these two techniques can be achieved in several ways depending on the application at hand [9]. 
Empirical Mode Decomposition (EMD) is a new powerful data analysis method for nonlinear and nonstationary signals. Being a data adaptive decomposition technique, EMD can help decompose any complex data set into zero mean oscillating components called IMF and the Residue itself being an IMF [10]. These IMFs give sharp and meaningful identification of instantaneous frequencies. The frequencies of these IMFs may overlap at any time instant and that these instantaneous frequencies different. We would like to stress that EMD is not a band pass technique but effective in decomposing nonlinear and non-stationary signals based on their local frequency characteristics. We explore this unique property of the EMD, to separate the noise inherent in the load data series. The local features will still be prominent in the IMFs and this makes it possible to observe the cycle, trend and random components in the time series.

An original technique was presented [11] for exploring long term load dynamics by use of multi-scale analysis of the daily peak load based on EMD. EMD is used to decompose the original load signal into intrinsic oscillatory components referred to as IMF (IMFs). Thereafter, physical interpretations and statistical description of the modes are discussed. The paper ends by comparing load components extracted by the EMD approach and that obtained from classical multiple linear regression models and concludes that, since EMD is a non-model based approach to seasonal adjustment of time-series, it tends to capture the changes in the structure of seasonal variation better.

EMD was applied [12] in forecasting the arrival time behaviors in a parallel Batch system. Using data of workload record, analysis reveals that IMF's products of the decomposition process of EMD yield better prediction values than original arrival histogram.

A method was proposed [13] combining EMD and Gene Expression Programming called EMD \& GEP. In the study, EMD is used to obtain the IMFS and the residual trend. By using corresponding load series of the same time but different days in the IMFS and residual trend as training samples, and by means of GEP, models of different time points in each IMF and residual trend are evolved based on time sharing. Final forecasting result is obtained by reconstruction of each of the IMFs and residual trend models. Results reveal that the proposed EMD \& GEP model is better when compared with wavelength and GEP models.

A novel short-term wind forecasting model [14] was put forward, based on ensemble empirical mode decomposition and combination of support vector machine. The methodology involves using both EMD and EEMD to identify intrinsic wind oscillatory modes but their characteristics time scale in the data empirically, and then decompose the wind speed series accordingly into different IMF with corresponding frequencies and the residue component. The final forecasting value is obtained by estimating the parameters that would determine each SVM model and finally reconstruct and concord all results forecasted by the SVM. The paper concludes that the newly proposed EEMD-SVM forecasting model outperforms all other models in terms of forecasting accuracy.

Wind power was identified [15] as having non-steady and non-periodic characteristics. Hence, EMD theory was employed to decompose the wind power time into random and trend components. After which, chaotic prediction is made for the random and trend components using neural network with radical basis function and least squares support vector machine respectively. The final consequence was obtained by combining the prediction result of each component. The study concludes that the proposed model prediction accuracy is higher when compared with traditional ANN prediction model.

Guo et al. [16] came up with a paradigm of modified empirical mode decomposition based feed-forward neural network ensemble learning model. EMD is used to decompose the nonlinear and non-stationary original wind speed series into a finite and small number of IMFs and one residue. According to them, it would give a deep insight into the data structure. This sub-series except the high frequency are forecasted respectively by FNN whose input variables are obtained by Partial Auto Correlation Function (PACF). The ensemble forecast is obtained by summing up the results of the model IMFS and residual series together. It was found out that the developed model obtained the best forecast accuracy when compared with basic FNN and unmodified EMDbased FNN.

In this paper we report novel combination model for Electric Peak Load Forecasting. In the proposed model, EMD data decomposition property is first utilized in decomposing the data into several IMFs that are stationary. These stationary IMFs are then employed in developing the model proposed in this study. Lastly the proposed model is used for forecasting purposes to test its forecasting accuracy ability. 
The remaining part of this paper is structured as follows: Section 2 is the methodology in which we discuss the EMD technique extensively followed by the discussion of ANFIS models. The section closes by discussing the proposed EMD-ANFIS model. Section 3 concerns itself with implementation of the proposed model on a real life data taken from a Power Holding Company of Nigeria (PHCN) Bida and discussion of results. The conclusion is presented in section 4 and Section 5 is acknowledgement in which we appreciated the contributions of few organizations towards the realization of this research work.

\section{Methodology}

In this section, we attempt to discuss the key components of the proposed forecasting method, that is, EMD-ANFIS. We first discuss the EMD extraction process extensively, then we discuss ANFIS model that will be combined with the EMD and lastly, we present a comprehensive description of the proposed model.

\subsection{Empirical Mode Decomposition}

The concept of EMD was stated by Haung et al [10]. Other studies have equally employed the technique [17-19]. In Load Forecasting, EMD has been implemented $[13,11]$.

EMD is suitable for processing time series data that are non-stationary and non-linear. The process by which the IMFS are extracted is referred to as sifting process [10].

EMD is a time-frequency analysis method [10]. It provides an alternative to conventional analysis such as Wavelength Transform. EMD assumes that the data has several coexisting modes of oscillations inherent in it. The main idea behind EMD is to decompose the signal in a given data into several signals that are stationary. These signals that are stationary are referred to as IMFs. The IMF and the residue (itself an IMF) contain components of different frequency bands from the highest to the lowest. IMF1 has the highest frequency and the frequencies decreases progressively along the line. This ensures that the characteristics of the load data can be revealed under different resolutions which are not the case in Wavelength Transform. Furthermore, in EMD, the original data can be reconstructed without information loss by adding the respective IMFS and the residue. The IMFs brings to the fore, the local characteristics inherent in the data and through analyzing the IMFs, the characteristic information of the original data can be obtained. To be an IMF, a function must meet the following two conditions: 1) the mean value of the upper envelope defined by the local maxima and the lower envelope defined by the local minima must be zero. 2) The number of the local maxima and the number of the local minima must differ by at most one.

\subsubsection{The EMD Algorithm}

Step 1: Identify the maxima and minima of the load data, $x(t)$.

Step 2: Construct the lower $\mathrm{e}_{\min }(\mathrm{t})$ and upper envelopes $\mathrm{e}_{\max }(\mathrm{t})$ by interpolating with cubic spline.

Step 3: Calculate the mean value by obtaining the average of the upper and lower envelopes

$$
m(t)=\frac{\left[e_{\min (t)}+e_{\max (t)}\right]}{2}
$$

Step 4: Subtract the mean from the original series

$$
d(t)=x(t)-m(t)
$$

Step 5: Check $d(t)$ properties

- If $d(t)$ is an IMF, as stated above, then denote it as ith IMF and replace $\mathrm{x}(\mathrm{t})$ with the residue $r(t)=$ $x(t)-d(t)$. The ith IMF is often denoted as $C_{i}(t)$ and $i$ is called its index [17].

- If it is not, replace $x(t)$ with $d(t)$.

Step 6: Repeat steps 1 to 5 until the stopping criterion is satisfied by the residue. The stopping criterion proposed by Huang et al. [20].

Iterate up to the pre-defined times after the residue has satisfied the restriction that the zero-crossings and the extrema do not differ by more than one, the sifting process can be stopped by any of the following criteria which must have been determined beforehand: either when the component or residue is so small that it is less than a pre-determined value of a substantial sequence or

a. when the residue becomes a monotonic function from which it is no longer possible to extract anymore IMFS or

The original data $x(t)$ can be represented as

$$
x(t)=\sum_{i=1}^{n} I M F_{i}+r(t)
$$

where, $n$ is the number of residues and $r(t)$ is the final residue.

The algorithm presented is shown in the Figure 1. 


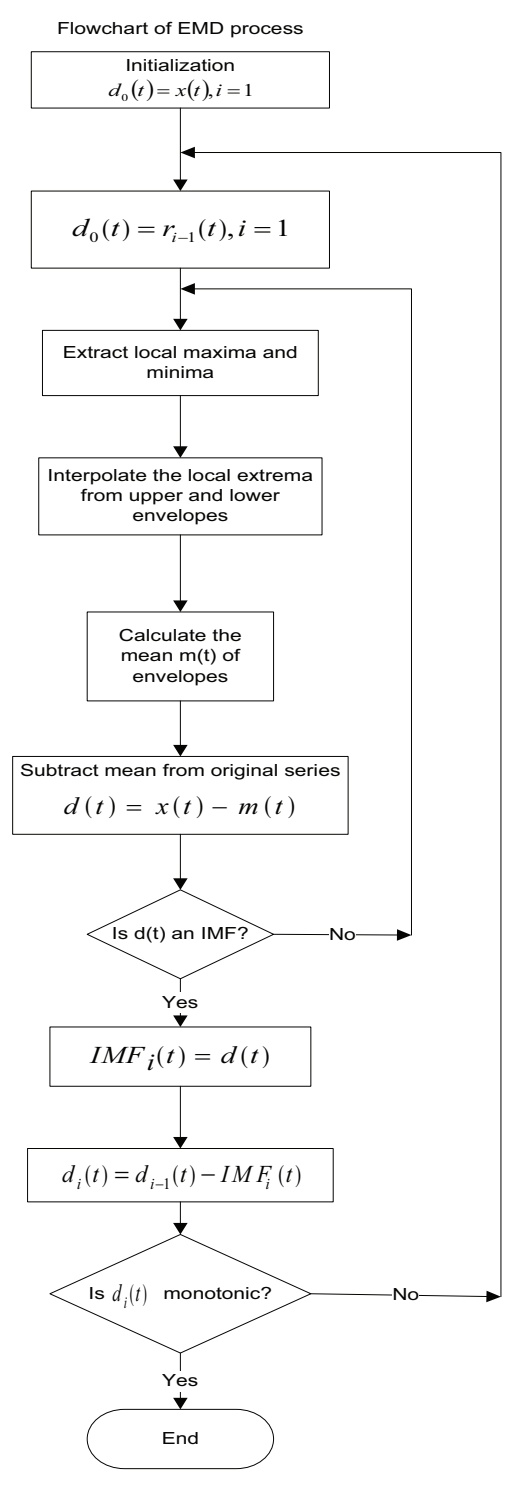

Figure 1. The EMD sifting process.

\subsection{Adaptive Neuro-Fuzzy Inference System (ANFIS)}

ANFIS is a hybrid model developed by Jang [21] in to overcome the inability of Fuzzy Inference System (FIS) to learn things. ANFIS combines the learning skills of ANN with the inference ability opinion of expert opinion based on FIS models. It adjusts the membership functions of all variables input and output inclusive and generates the appropriate input and output rules automatically. ANFIS can extract all rules in the dataset and experts are left with just the task of interpreting the rules.

Consider two inputs $x, y$ and one output $z$. Figure 2 gives a typical ANFIS structure.

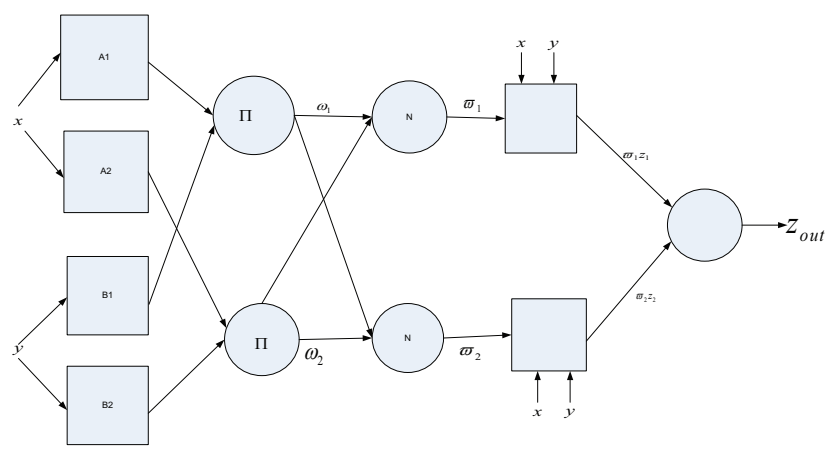

Figure 2. A typical ANFIS structure.

LAYER 1: Every node in this layer is an adaptive node with node function as:

$$
\begin{gathered}
O_{1, i}=\mu_{A_{i}}(x) \text { for } i=1,2 \\
O_{1, i}=\mu_{B_{i-2}}(y) \text { for } i=3,4
\end{gathered}
$$

where, $x$ (or $y)$ is the input of the node, $A_{i}\left(\right.$ or $\left.B_{j}\right)$ is the linguistic label, $\mu(x)(\operatorname{or} \mu(y))$ is the membership function, usually adopting the bell shape with maximum and minimum equal to 1 and 0 , respectively, as follows:

$$
\mu(x)=\frac{1}{1+\left(\frac{x-c_{i}}{a_{i}}\right)^{2 b_{i}}}
$$

where, $\left\{a_{i}, b_{i}, c_{i}\right\}$ is the parameter set. As the values of these parameters change, the bell shaped functions vary accordingly. The parameters in this layer are named premise parameters.

LAYER 2: Every node in this layer is a fixed node, marked by a circle and labeled $\pi$, with the node function to be multiplied by input signals to serve as output signal.

$$
O_{2, i}=\mu_{A_{i}}(x) \cdot \mu_{B_{i}}(y) \quad \text { for } \quad i=1,2
$$

The output signal represents the firing strength of the rule.

LAYER 3: All nodes in this layer are fixed. Marked by a circle and labeled $\mathrm{N}$, its function is to normalize the firing strength by calculating the ratio of the ith node firing strength to the sum of all rules' firing strength.

$$
O_{3, i}=\frac{\omega_{i}}{\sum \omega_{i}}=\frac{\omega_{i}}{\omega_{1}+\omega_{2}}=\bar{\omega}_{i} \quad \text { for } \quad i=1,2
$$

LAYER 4: The nodes in this layer are adaptive, marked by a square and the node function is 


$$
O_{4, i}=\bar{\omega}_{i} \cdot z_{i} \quad \text { for } \quad i=1,2
$$

where, $Z_{1}$ and $Z_{2}$ are the fuzzy if-then rules as stated below

RULE 1: if $x$ is $\mathrm{A}_{1}$ and $y$ is $\mathrm{B}_{1}$ then

$$
z_{1}=p_{1} x+q_{1} y+r_{1}
$$

Rule 2: if $x$ is $\mathrm{A}_{2}$ and $y$ is $\mathrm{B}_{2}$ then

$$
z_{2}=p_{2} x+q_{2}+r_{2}
$$

where, $\left\{p_{i}, q_{i}, r_{i}\right\}$ is the parameter set, referred to as the consequent parameters.

LAYER 5: Every node in this layer is fixed, marked by a circle and labeled $\Sigma$, with node function for overall output given by

$$
O_{5}=\sum_{i} \bar{\omega}_{i} \cdot z_{i}=z_{\text {out }}
$$

Suppose the training data has $\mathrm{n}$ entries, and then the overall error measure will be given by

$$
E=\sum_{i=1}^{n} E_{i}=\sum_{i=1}^{n}\left(R_{i}-f_{\text {outi }}\right)^{2}
$$

where, $E_{i}$ is the error measure for the ith entry of the given training data set, $R_{i}$ is the desired output of the ith entry and $Z_{\text {out }}$ is the output of the ANFIS using the ith entry.

\section{EMD-ANFIS Model}

The process to achieve this is outlined in the steps below.

Step 1: the historical load data is passed through the EMD extraction process described in 2.1 above and by doing this several IMFs is obtained.

Step 2: Since the ANFIS model requires feeding the network with target, this too must undergo the EMD extraction process. Here, the maximum and minimum values of the targets must be adjusted severally to ensure that the same number of IMFs is obtained as those in step 1 above. For instance, if step1 has 9 IMfs step 2 must be adjusted so that the EMD process yields 9IMFs also.

Step 3: the IMFs for the load which is the input data for the network is normalized likewise the target. In this paper all inputs and all targets were normalized to a scalable range of between 0 and 1 . This was achieved by means of the following:

$$
X_{\text {norm }}=\frac{X_{i}-X_{\min }}{X_{\max }-X_{\min }}
$$

Step 4: with an IMF from the input and its corresponding target IMF. The EMD-IMF models are developed, that is IMF1 for input is taken alongside IMF1 for target to develop the first model. Likewise IMF2 for input with IMF2 for target is used to develop the second model and so on until all the IMFs for both input and target have been used.

Step 5: Combine all results obtained from step 4 together. Finally, the results are transformed from their normalized state back to their original form. This is achieved by manipulating equation (14) as follows:

$$
X_{\text {Normalised }} \times\left(X_{\max }-X_{\min }\right)+X_{\text {min }}
$$

This combine results is the proposed EMD-ANFIS model and can then be used for forecasting purposes.

\section{Results and Discussion}

The paper adopts the Daily Peak Load Demand (DPLD) of Power Holding Company of Nigeria, Bida from January $1^{\text {st }}, 2012$ to December $12^{\text {th }}, 2012$ for modeling and testing by applying the methods described above. $70 \%$ of the data was used for training the network while 30\% were used for testing. All analyses were implemented using MATLAB. Figure 3 show the 8 IMFs and residue components.

Figure 4 and Figure 5 are the forecasting results obtained by the EMD-ANFIS model developed.

For evaluating the performance of the model, we adopted the measure, Mean Absolute Percentage Error (MAPE). The index is presented as follows:

$$
M A P E=\frac{1}{N} \sum_{i=1}^{N}\left|\frac{Y_{i(\text { actual })}-Y_{i(\text { forecast })}}{Y_{i(\text { actual })}}\right|
$$

In the index above, $Y_{i \text { (actual) }}$ is the actual time series for period $i, Y_{i \text { (forecast) }}$ is the forecast time series for period $i$, while $\mathrm{N}$ is the total number of forecast. We equally took the weekday and weekend values of the time series and implemented the EMD-ANN technique on these still considering $70 \%$ as training data and $30 \%$ as testing data set. The results are presented in Table 1; Figure 6 and Figure 7.

The EMD-ANFIS model yields the best results for all three models under consideration, which are whole year, weekday and weekend models. This is an indication that the proposed EMD-ANFIS model is suitable for all types 
Table 1. MAPE for all three models

\begin{tabular}{lccc}
\hline Type of model & EMD-ANFIS & EMD-ANN & $\begin{array}{l}\text { Traditional } \\
\text { ANN }\end{array}$ \\
\hline Whole Year Model & 2.4997 & 2.5145 & 5.00 \\
Weekday Model & 2.4999 & 2.5093 & 5.01 \\
Weekend Model & 2.4998 & 2.5011 & 5.01 \\
Weighted Average & $\mathbf{2 . 4 9 9 8}$ & $\mathbf{2 . 5 1 0 8}$ & $\mathbf{5 . 0 0 5 0}$ \\
\hline
\end{tabular}

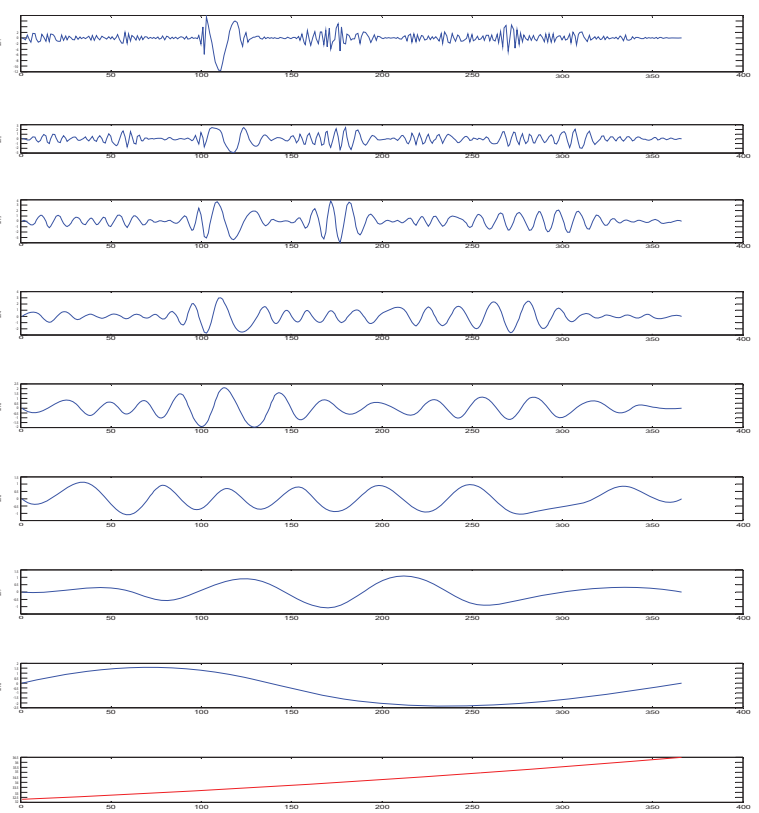

Figure 3. Extracted IMFs and residue.

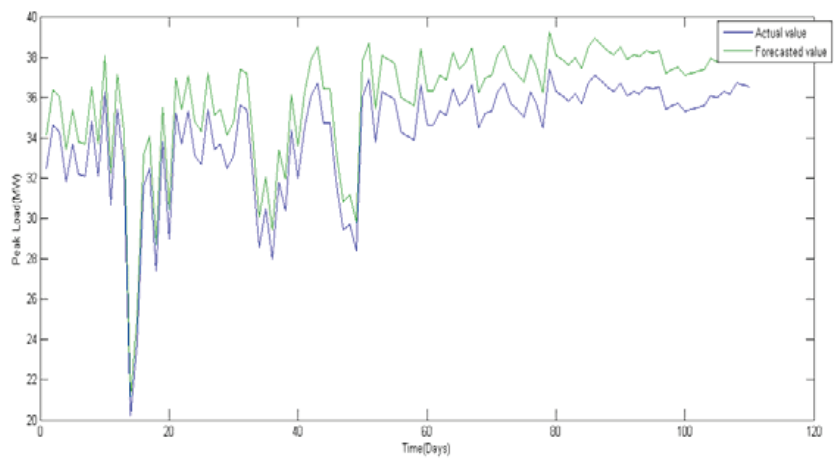

Figure 4. Actual and forecasted data for whole year (one day ahead forecast).

of models in the ESI. Due to non-availability of data we took $5 \%$ of our actual data as target, since the standard practice in ESI is to set as next near target $5 \%$ of previous year's load, and because the proposed model is very adaptive it tends to follow this pattern perfectly.

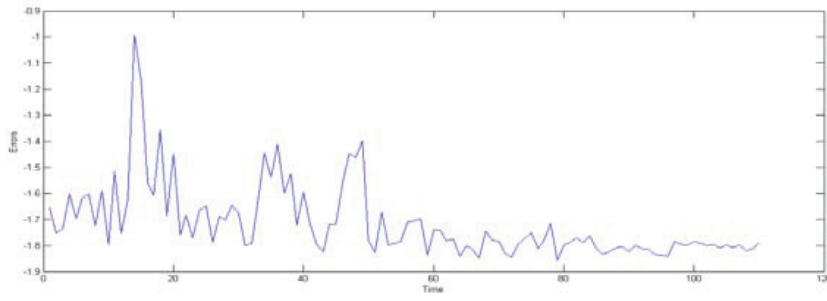

Figure 5. Error curve for whole year model (one day ahead forecast).

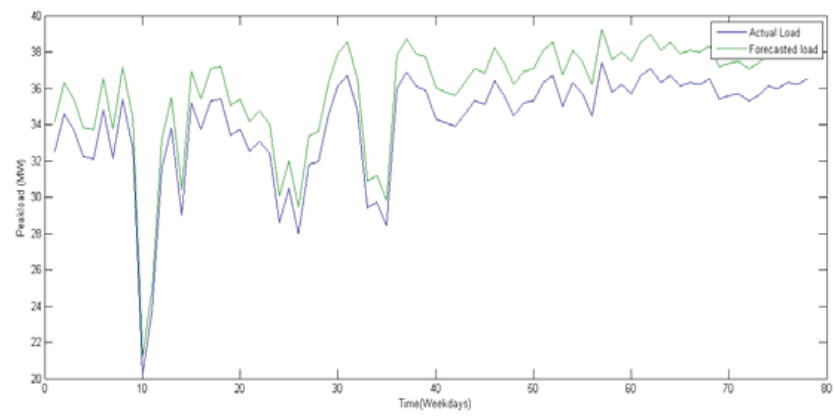

Figure 6. Actual and forecasted data for weekday model (monday to friday) load.

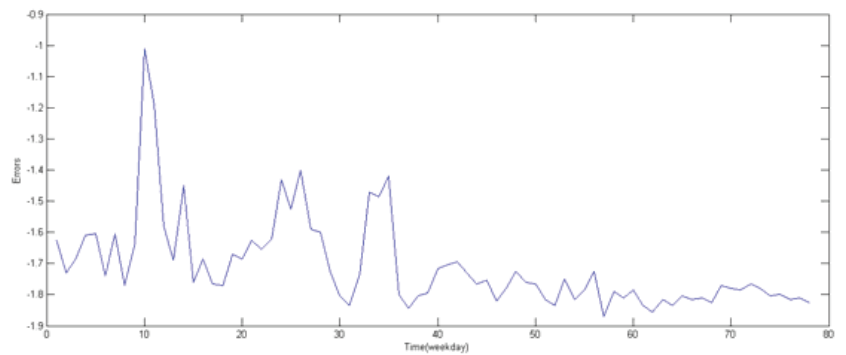

Figure 7. Error curve for weekday forecast result.

It can also be observed from the results presented in Table 1 that the EMD-ANFIS model yields better forecasting results when compared with the traditional ANN and by this, infer that EMD is a good data preprocessing method for time series modeling and that the EMD-ANFIS model is effective model for load forecasting in ESI. Further it reveals that the EMD-ANFIS model improves the performance of EMD-ANN model by $2.76 \%$ while it improves the performance of traditional ANN model by $50.05 \%$.

\section{Conclusion}

In this paper, we use a method based on EMD and ANFIS to establish a model for Electric Peak load demand forecasting. The EMD is used to decompose the load data 
into 8 stationary IMFs and a residue component. Then appropriate ANFIS models were developed for each of these. Finally, we combined the results of the various models, and with this we developed the EMD-ANFIS model. The EMD-ANN model presented in this paper yielded better forecasting results when compared with EMD-ANN and traditional ANN models.

\section{Acknowledgement}

The authors would like to thank the Ministry of Higher Education (MOHE), Malaysia for the support received for the implementation of this study by providing funds through grant with vote number $02 \mathrm{H} 52$ for this research work. We would also like to appreciate the contributions of the management of Universiti Teknologi, Malaysia for IDF scholarship award to one of the authors of this paper and for allowing the use their facilities for this study. Lastly, we would like to thank the management of Power Holding Company of Nigeria ( $\mathrm{PHCH})$ Bida for providing the data for this study.

\section{References}

1. WenXiao M, XiaoMin B et al. (2002). Short-term load forecasting with artificial neural NETWORK and fuzzy LOGIC, International Conference on Power System Technology, vol 2, 1101-1104.

2. Thammano A (1999). A new forecasting approach with neuro-fuzzy architecture, Systems, Man, and Cybernetics, IEEE SMC '99 Conference Proceedings, 1999 IEEE International Conference on, vol 1, I-386-I-389.

3. Holmukhe R M, Dhumale S et al. (2010). Short-term load forecasting with fuzzy Logic systems for power system planning and reliability-a review, AIP Conference Proceedings, vol 1298(1), 445-458.

4. Jain A, and Satish B (2008). Integrated approach for shortterm load forecasting using SVM and ANN, TENCON-2008 IEEE Region 10 Conference, 1-6.

5. Selakov A, LLic S et al. (2012). A comparative analysis of SVM and ANN based hybrid model for short-term load forecasting, 2012 IEEE Transmission and Distribution Conference and Exposition, 1-5.

6. Liao G C (2006). An application of fuzzy neural network combined with chaos genetic algorithm and simulated annealing to short-term load forecasting, IEEE Transaction on Evolutionary Computation, vol 10(3), 330-340.

7. Ling S H, Frank H et al. (2003). A novel genetic-algorithmbased neural network for short-term load forecasting, IEEE Transaction on Industrial Electronics, vol 50(4), 793-798.
8. Denai M A, Palis F et al. (2004). ANFIS based modelling and control of non-linear systems: a tutorial, 2004 IEEE Conference on Systems, Man and Cybernetics, vol 4, 3433-3438.

9. Jang J S R, Sun C T et al. (1997). Neuro Fuzzy and SoftComputing, Prentice Hall, New Jersey.

10. Huang N E, Shen Z et al. (1998). An empirical mode decomposition and the hilbert spectrum for nonlinear and non-stationary time series analysis, Proceedings of the Royal Society of London, A, vol 454(1971), 903-995.

11. Mahmoud M M, Mhamdi F et al. (2009). Long-term multi-scale analysis of the daily peak load based on empirical mode decomposition, 2009 IEEE Bucharest Power Tech Conference, 1-6.

12. Ngo L, Apon A et al. (2010). A forecasting capability study of empirical mode decomposition for the arrival time of a parallel batch system, 2010 IEEE Seventh International Conference on Information Technology, 420-425.

13. Fan X, and Zhu Y (2010). The application of empirical mode decomposition and gene expression programming to short-term load forecasting, IEEE Sixth International Conference on Natural Computation, vol 8, 4331-4334.

14. Jia S (2011). A new method for the short-term wind speed forecasting, IEEE $4^{\text {th }}$ International Conference on Electric Utility deregulation and Restructuring and Power Technologies, 1320-1324.

15. Zhang Y, Lu J et al. (2012). Wind power short-term forecasting based on empirical mode decomposition and chaotic phase space reconstruction, Journal of Automation of Electric Power Systems, vol 5, 24-28.

16. Guo Z, Zhao W et al. (2012). Multi-step forecasting for wind speed using a modified EMD-based artificial neural network model, Journal of Renewable Energy, vol 37(1), 241-249.

17. Zhang X, Lai K K et al. (2008). A new approach for crude oil price analysis based on empirical mode decomposition, Energy Economics, vol 30(3), 905-918.

18. Blanco-Velasco M, Weng B et al. (2008). ECG signal denoising and baseline wander correction based on the empirical mode decomposition, Computers in Biology and Medicine, vol 38(1), 1-13.

19. Demir B, and Erturk S (2008). Empirical mode decomposition pre-process for higher accuracy hyperspectral image classification, Geoscience and Remote Sensing Symposium, IGARSS 2008 IEEE International, vol 2, II-939-II-941.

20. Huang N E, Wu M L et al. (2003). A confidence limit for the empirical mode decomposition and hilbert spectrum analysis, Proceedings of the Royal Society of London, A, vol 459(2037), 2317-2345.

21. Jang J-S (1993). ANFIS: adaptive-network-based fuzzy inference system, Systems, Man and Cybernetics, IEEE Transactions on, vol 23(3), 665-685. 